\title{
Chirurgische und Interventionelle Behandlungsmöglichkeiten beim Adenokarzinom des Pankreas
}

Mathias Worni, MD MHS, Beat Gloor, MD

Universitätsklinik für Viszerale Chirurgie und Medizin, Universität Bern, Inselspital, 3010 Bern. mathias.worni@insel.ch

Bei nur einer Minderheit von 20\% der Patienten mit Pankreaskarzinom wird die Erkrankung in einem noch lokal begrenzten Stadium diagnostiziert, das eine primäre onkologisch radikale Resektion erlaubt, womit ein 5-Jahres Überleben von 20 bis 25\% erreicht wird. Aber auch bei sogenannt „borderline resectable“ und "locally advanced" Befunden kann eine chirurgische Behandlung eventuell kombiniert mit intraoperativer irreversibler Electroporation in ausgewählten Situationen und im Rahmen von kombinierten Behandlungskonzepten eingesetzt werden, was die Prognose deutlich verbessert. In high-volume Zentren mit entsprechender Erfahrung können auch bei Tumoren mit begrenzter Infiltration der V.portae/V.mesenterica superior durch Venenresektion und -rekonstruktion radikale Resektionen erreicht werden. 


\section{Hintergrund:}

Das Adenokarzinom des Pankreas ist mit ca. 1,200 Neuerkrankungen in der Schweiz eine häufige Erkrankung.[1, 2]. Eine Mehrheit ( 80-95\%) der Erkrankten wird auch an den Folgen des Pankreaskarzinoms sterben. Die chirurgische Resektion des Tumors ist die einzige Therapieoption, welche eine Chance auf Heilung bringt. Leider ist die Operation in nur ca. 20\% der Patienten möglich, da sich die anderen Patienten bereits mit einem fortgeschrittenen Tumorleiden präsentieren. Bei $50 \%$ der Neudiagnosen werden bei der Erstdiagnose Metastasen gefunden während in rund 30\% der Fälle die Patienten ein lokal fortgeschrittenes Tumorleiden aufweisen, welches eine vollständige chirurgische Resektion unter vertretbaren Risiken nicht erlaubt[3]. Das 5-Jahres Überleben nach kombinierter Behandlung mit vollständiger operativer Tumorentfernung erreicht 20-25\% [4]. Die Gründe für diese schlechte Prognose sind noch nicht vollständig geklärt. Im Vordergrund stehen die ChemotherapieResistenz, das frühe Auftreten von Mikrometastasen sowie eine häufig mikroskopisch nicht vollständige chirurgische Entfernung des Tumors[5].

\section{Abklärung - Staging}

Beim Pankreaskarzinomen fehlen Marker und Frühsymptome (falls der Tumor nicht auf Grund der Lokalisation früh zu einem schmerzlosen Ikterus führt).Der Verdacht auf ein Pankreaskarzinom wird häufig erst erhoben nach der Durchführung einer Computertomographie wegen unspezifischen Abdominalbeschwerden, bei ungeklärtem Gewichtsverlust oder zunehmenden abdominellen oder Rücken-Schmerzen [6]. Zur genauen radiologischen Darstellung des Primärtumors reicht meist eine Computertomographie mit Kontrastmittel-Darstellung der arteriellen und portalvenösen Gefässe[7]. In unklaren Situationen sind die Magnetresonanztomographie (MRI) mit MagnetresonanzCholangiopankreatographie (MRCP) sowie der zunehmend eingesetzte endoskopische Ultraschall (EUS) sehr hilfreich. Die Endoskopie hat zudem den Vorteil, dass gleichzeitig eine endoskopische retrograde Cholangiopankreatographie (ERCP) mit Stenteinlage zur Sicherstellung des Galleabflusses oder eine Feinnadelpunktion des vermuteten Tumors durchgeführt werden können. Ausserdem besteht die Möglichkeit, bei signifikantem Gewichtsverlust des Patienten, auch gleich eine nasojejunale Sonde zur präoperativen Ernährung einzulegen. Welche diagnostischen Modalitäten zur Diagnosesicherung vor Therapiebeginn durchgeführt werden sollen ist abhängig von der Ausdehnung des Tumors, der klinischen Präsentation des Patienten, sowie des zukünftigen Behandlungsplans.

Neben der gängigen TNM Klassifikation maligner Tumore werden Pankreaskarzinome bezugnehmend auf die therapeutischen Konsequenzen eingeteilt. Dabei unterscheidet man basierend auf den Resultaten der Bildgebung zwischen: 
- primär resezierbaren,

- „borderline resectable“,

-,locally advanced“ sowie

- bereits metastasierten Leiden[8]. Während die Gruppe der primär resezierbaren und bereits metastasierten Pankreaskarzinomen meist klar definiert werden kann, ist die Einteilung in „borderline resectable" oder "locally advanced" nicht immer einfach. Es existieren unterschiedliche Definitionen für diese beiden Stadien. Bei Patienten mit „borderline resectable disease" ist die Beteiligung der umliegenden Gefässe geringer als bei "locally advanced disease", eine Resektion und Rekonstruktion der Venenachse ist möglich während die begrenzte Beteiligung der Arteria hepatica respektive Arteria mesenterica superior eine Resektion derselben nicht notwendig macht[9] Patienten mit einem "borderline resectable“ Tumorstadium profitieren meist von einer chirurgischen Resektion, da die beteiligten Gefässe erhalten respektive sinnvoll ersetzt werden können[10]. Im Gegensatz profitieren Patienten mit "locally advanced disease" meist nicht von einer Operation, weil eine komplette Tumorentfernung nur selten möglich ist, weil das perioperative Risiko zu hoch ist und sich das Überleben gegenüber nicht resezierten Patienten nicht verbessert[11].

Eine histo-pathologische Diagnosesicherung bei radiologisch eindeutigen Zeichen eines resezierbaren Pankreaskarzinoms ist vor einer operativen Resektion nicht zwingend notwendig [12]. Im Gegensatz dazu wird diese aber vor Durchführung einer (Radio-) Chemotherapie gefordert. Bei unklarem Lokalbefund respektive bei radiologischem Verdacht auf „locally advanced disease" wird mittels endoskopischem Ultraschall biopsiert. Falls trotz mehrfachem Versuch eine affirmative Diagnose nicht gestellt werden kann, wird der Tumor auch selten laparoskopisch oder gar offen transduodenal biopsiert. Durch diesen Zugang können deutlich grössere Biopsienadeln verwendet werden und die Trefferwahrscheinlichkeit steigt signifikant an. Bei radiologischem Verdacht auf Lebermetastasen ist es ausreichend, diese - z.Bsp. ultraschallgesteuert - zu biopsieren.

\section{Chirurgie}

Alle Patienten ohne nachgewiesene Fernmetastasierung sollten nach erfolgtem Staging sowie unter Einbezug des Hausarztes und nach Rücksprache mit dem Patienten an einem interdisziplinären Tumorboard zur Therapieplanung besprochen werden. Der Entscheid für oder gegen eine primäre Operation, ein neo-adjuvantes Therapiekonzept oder eine Durchführung einer lokal ablativen Therapie (z.Bsp. mit irreversibler Elektroporation, siehe unten) ist komplex und muss neben technischen Möglichkeiten immer auch die Leistungsfähigkeit des Patienten sowie die zu erwartenden Auswirkungen auf die 
Lebensqualität berücksichtigen. Ein erster Entscheid ist jeweils zu fällen, ob die geplante Karzinomtherapie in kurativer oder nicht-kurativer Absicht empfohlen wird. Dabei ist die Chirurgie die aktuell einzige Therapiemodalität, welche eine Heilung des Tumorleidens erzielen kann. [4]

Falls eine chirurgische Resektion möglich ist, definiert die Lokalisation des Pankreaskarzinoms die Art sowie das Ausmass der Pankreasresektion. Ist ein Tumor hauptsächlich im Pankreaskopf gelegen, erfolgt die Resektion meist als pylorus-erhaltende oder klassische Whipple Operation (Abbildung 1A). Eine solche Operation kann auch Richtung Pankreasschwanz erweitert werden, falls dies die Ausdehnung des Tumors in der Bauchspeicheldrüse erfordert. Ist der Tumor im Pankreasschwanz gelegen, erfolgt eine Pankreas-Linksresektion inklusive Splenektomie (Abbildung 1B). Diese Operation kann in Analogie zur Whipple-Operation falls notwedig nach rechts erweitert werden. Selten indiziert aber manchmal notwendig ist eine totale Pankreatektomie. Resektionen der Venenachse bei Verdacht auf deren Infiltration sind ohne prohibitiven Anstieg der perioperativen Morbidität und Mortalität möglich während gleichzeitige arterielle Rekonstruktionen nur in Ausnahmesituationen durchgeführt werden sollten.[9, 13] Limitierte Resektion wie PankreasSegmentresektionen oder Enukleationen sind aus onkologischer Sicht, insbesondere wegen der Notwendigkeit der Lymphknotenentfernung nicht sinnvoll. Während die Pankreaslinksresektion inklusive Splenektomie mit sehr guten Resultaten laparoskopisch durchführbar ist, hat sich die laparoskopische Whipple-Operation bisher noch nicht durchgesetzt[14, 15]. Diese ist technisch sehr anspruchsvoll und ein Vorteil gegenüber der offenen Operation konnte noch nicht überzeugend gezeigt werden.

Der Stellenwert von palliativen Interventionen hat sich in den letzten Jahren Richtung endoskopischer oder perkutaner Interventionen verlagert. Die Möglichkeiten mittels Stents den Verschlussikterus auch langfristig zu beheben sind heutzutage sehr gut und ein Umstieg auf eine chirurgisch angelegte Hepatiko-Jejunostomie ist nur in speziellen Ausnahmefällen notwendig. Patienten mit einer tumorbedingt erschwerten per oralen Nahrungsaufnahme durch pyloro-duodenale Obstruktion können von einem intraluminalen Stent oder einer laparoskopischen Gastro-Jejunostomie profitieren. Dies ermöglicht den Patienten meist, sich wieder per oral ernähren zu können mit deutlicher Verbesserung der Lebensqualität.

\section{Postoperative Auswirkungen und Nachsorge}


Pankreaschirurgie bei Pankreaskarzinomen bedarf eines interdisziplinär eingespielten und erfahrenen Teams. Die Zentralisierung von komplexen Operationen inklusive der Pankreaschirurgie im Rahmen der hochspezialisierten Medizin (HSM) auf ausgewählte Spitäler hat zum Ziel die Qualität zu verbessern und damit die postoperative Morbidität und Mortalität zu reduzieren sowie die Langzeitprognose der Patienten zu verbessern. In weltweit unabhängig durchgeführten Studien konnte immer wieder gezeigt werden, dass eine erhöhte Fallzahl pro Spital und Chirurg zu einer deutlichen Reduktion der postoperativen Mortalität führt[16-18]. Das Ziel eines jeden Zentrums für Pankreaschirurgie sollte sein, eine perioperative Mortalität bei Pankreaskarzinomoperationen von <5\% zu erzielen. (Abbildung 2).

Je nach Ausmass der präoperativen Pankreasstauung und der daraus resultierenden Pankreasatrophie sind die exo- und endokrine Funktion schon präoperativ reduziert. Wird nun noch ein relevanter Anteil an Bauchspeicheldrüse entfernt, akzentuiert sich eine exokrine und/oder endokrine Pankreasinsuffizienz.

Regelmässige Kontrollen der Blutzuckerwerte sind nach Pankreasresektionen auch bei initial normalen Blutzuckerwerten empfehlenswert.

Um postoperativ die Auswirkungen einer exokrinen Pankreasinsuffizienz zu minimieren, werden am Inselspital alle Patienten nach Resektionen der Bauchspeicheldrüse mit Pankreasenzympräparaten substituiert (z.Bsp. Creon, Panzytrat). Die Messung der Stuhlelastase ohne Notwendigkeit des Absetzens der Enzympräparate erlaubt im Verlauf eine Quantifizierung der exokrinen Eigenleistung der Bauchspeicheldrüse und Anpassung respektive Sistierung der Substitutionstherapie.

\section{Multimodale Therapieansätze}

Liegt die genaue histopathologische Beurteilung (Tumortyp und Stadium) des Resktionspräparates vor werden diese Resultate zusammen mit allen anderen Information über den Patienten postoperativ erneut an einem interdisziplinären Tumorboard diskutiert. Meist - falls keine Kontraindikationen zu einer adjuvanten Therapie bestehen - wird eine adjuvante Chemotherapie mit Gemzitabine empfohlen. Die Kombination mit Bestrahlung wird in der Schweiz und in Europa im Gegensatz zu den USA nur selten empfohlen, da es keine guten Resultate für deren Wirksamkeit gibt. Studien zu alternativen adjuvanten ChemotherapieRegimes sind aktuell im Gange und untersuchen unter anderem die Wirksamkeit von mFOLFIRINOX (NCT01526135), FOLFOXIRI (NCT02355119) sowie die Kombination von NabPaclitaxel mit Gemcitabine (NCT01964430). 
Wegen der verbesserten Ansprechrate des Pankreaskarzinoms auf neue ChemotherapieRegimens wie FOLFIRINOX hat in ausgewählten Fällen die neo-adjuvante Chemotherapie bei lokal fortgeschrittenen Tumoren zu einem Downstaging geführt, dass eine Resektion im Verlauf doch möglich wurde.[19, 20] Die Lebenserwartung bei erfolgreich resezierten Tumoren entspricht in den bisher veröffentlichten Publikationen in etwa der Lebenserwartung von primär resezierten Pankreaskarzinomen. Welche Patienten für eine solche Strategie qualifizieren ist aktuell noch nicht abschliessend geklärt, verspricht aber in Zukunft auch solche Patienten operieren zu können, welche bisher nur (Radio-) Chemotherapie erhalten haben.

\section{Lokal ablative Verfahren / Irreversible Elektroporation}

In den letzten Jahren etablieren sich zunehmends lokal ablative Verfahren auch bei Pankreaskarzinomen.[21] Alle thermalen Ablationsverfahren haben jedoch einen limitierten Anwendungsbereich in der Nähe der Gefässe (Gefahr von Thrombosen und Blutungen und verminderte Wirkung durch den „heat sink effect“).

Der thermalen Ablation gegenüber steht die irreversible Elektroporation (IRE), ein Verfahren zur lokalen Behandlung von Weichteiltumoren, das erstmals 2009 bei lokal fortgeschrittenem Pankreaskarzinom angewendet wurde.[22] IRE arbeitet nicht mit Hitze/Kälte sondern mit kurzen,r sehr hohen Stromstössen zwischen mindestens zwei eingebrachten IRE-Nadeln. Der applizierte Strom bewirkt, dass in einem vordefinierten Volumen Zellen durch Nanoporen in der Zellmembran irreversibel geschädigt werden. Der daraus resultierende Kalzium-Einstrom lässt die Zellen apoptotisch Zugrunde gehen.[23] Weil die Zellen durch Apoptose zerstört werden und nicht durch thermal induzierte Nekrose können Arterien und Venen sowie der Gallen- und Pankreasgang geschont werden. Diese Strukturen sind hauptsächlich aus Elastin und Kollagen aufgebaut und werden durch IRE nicht zerstört.[24] Zudem besteht bei der IRE kein „heat sink effect" und entsprechend ist die Methode auch sehr effektiv in der Nähe von Arterien und Venen, welche wegen ihrer Nähe zum Tumor häufig der limitierende Faktor sind für eine chirurgische Resektion oder thermale Ablation. Post-interventionelle Komplikationen sind relativ selten und beschränken sich meist auf gastrointestinale Komplikationen wie Blutung, Ulzerationen oder Gallengangsstrikturen während IRE-assoziierte Todesfälle sehr selten sind.[25, 26]

In einer Propensity-Score matched Studie an Patienten mit lokal fortgeschrittenen Pankreaskarzinomen waren das „local progression free survival“ (14 versus 6 Monate), das 
"distant progression free survival" (15 versus 9 Monate) als auch das Gesamtüberleben (20 versus 13 Monate) bei Patienten welche IRE erhalten haben signifikant besser als bei Patienten, welche diese Therapie nicht erhalten haben.[27] Zusätzlich fielen die Schmerzen auf der visuellen Analogskala im Mittel von 5/10 auf 3/10 . In der aktuell grössten Studie mit insgesamt 200 Patienten zeigten nach einem mittleren Follow-up von 29 Monaten nur 29\% der Patienten ein erneutes Auftreten des Tumors.[25] Das mittlere Überleben war mit 24.9 Monaten fast doppelt so lange wie das mittlere Überleben von Patienten, welche mit moderner Chemotherapie behandelt werden. Neben der Behandlung von Patienten mit lokal fortgeschrittenem Pankreaskarzinom wird zunehmend IRE auch intraoperativ angewendet, wenn ein positiver Resektionsrand auf Grund der präoperativen Bildgebung vermutet werden muss („margin accentuation IRE“). Die IRE hilft in diesen Situationen den Resektionsrand zusätzlich zu behandeln und eine komplette Tumorresektion zu ermöglichen. Vergleichende Langzeit-Daten von Patienten mit und ohne „margin accentuation IRE“ liegen aktuell noch nicht vor.

Die Platzierung der IRE-Nadeln ist einer der schwierigsten und wichtigsten Schritte zur Durchführung einer präzisen und erfolgreichen IRE-Behandlung. Am Inselspital in Bern wird diese Therapie in Zusammenarbeit mit ARTorg (Center for Biomedical Engineering Research of the University of Bern) mit dreidimensionaler computerbasierter Navigation unter kontinuierlicher Ultraschallkontrolle durchgeführt (Abbildung 3). Die Anwendung von Navigationshilfen zur Durchführung von lokal ablativen Therapien hilft, die Therapie sicher, zuverlässig und reproduzierbar durchzuführen.[28] 
Abbildung 1: Pankreaschirurgie - Resektionsausmass der typischen Operationen bei Pankreaskarzinom (A: Pankreaskopfkarzinom - pylorus-erhaltender (a) respektive klassischer (b) Whipple; B: Pankreasschwanzkarzinom - distale Pankreatektomie mit Splenektomie)

A
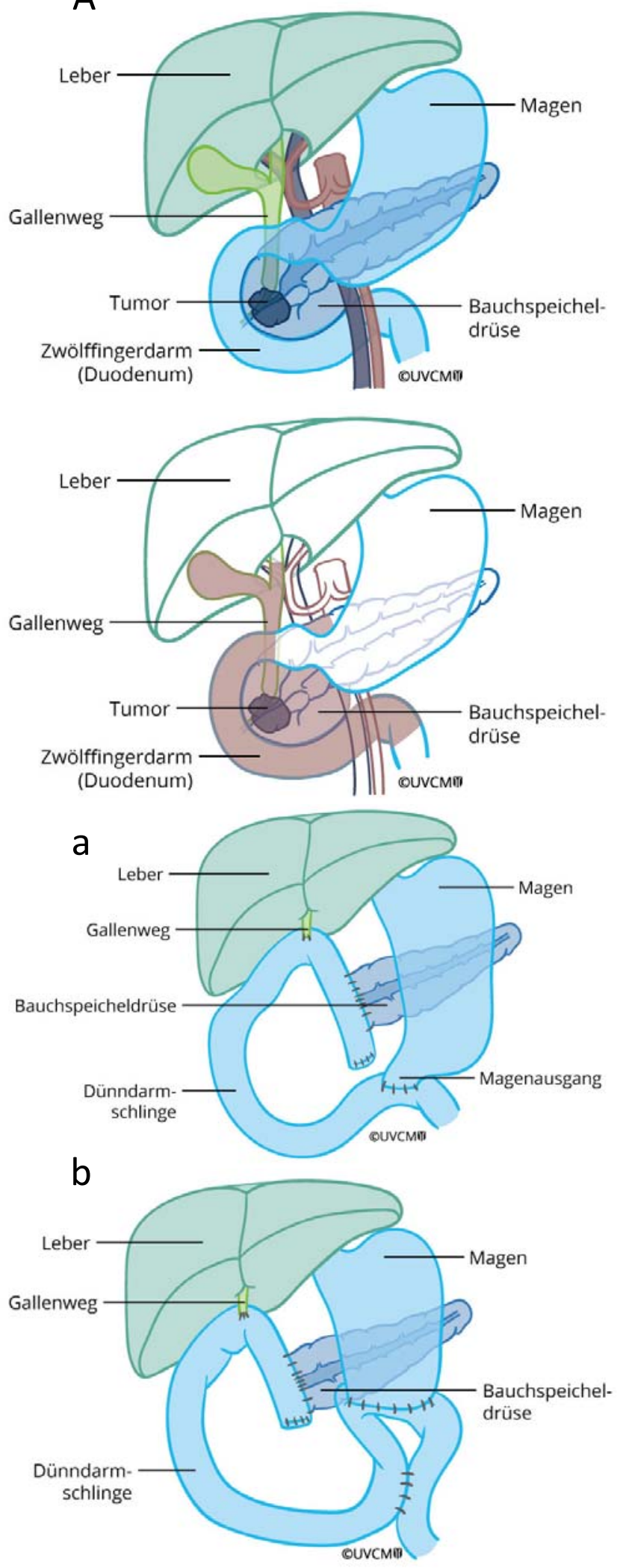

B
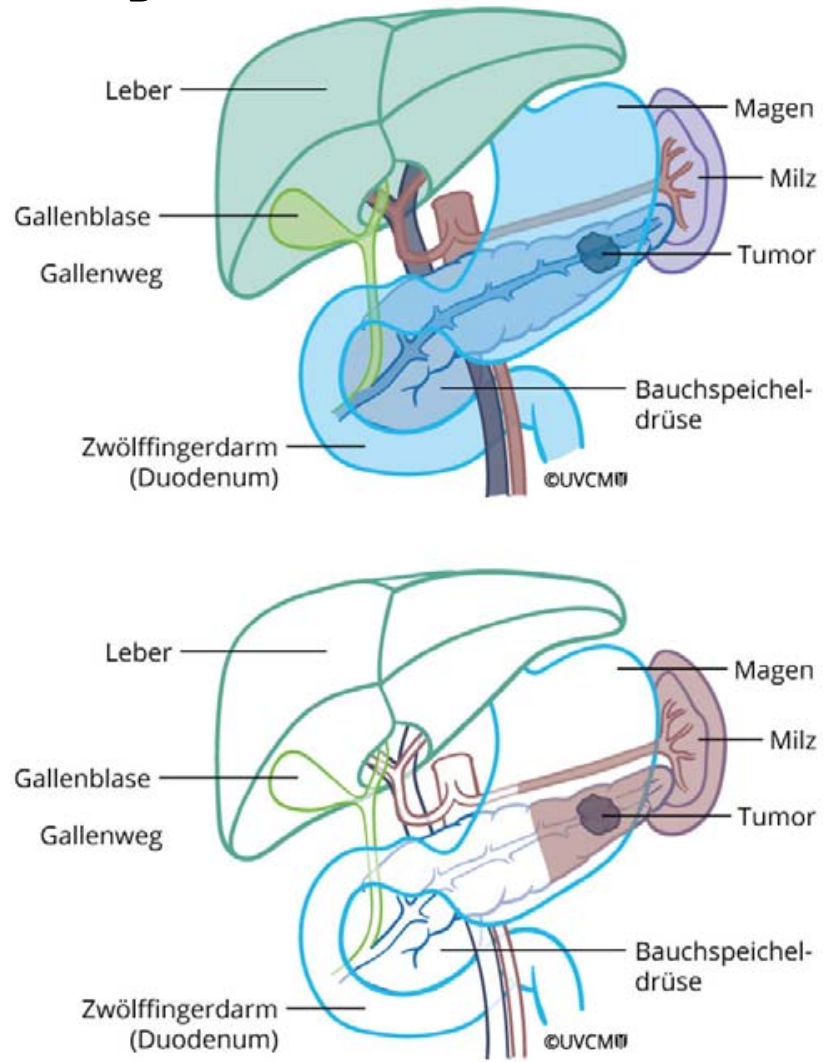

(Duodenum)

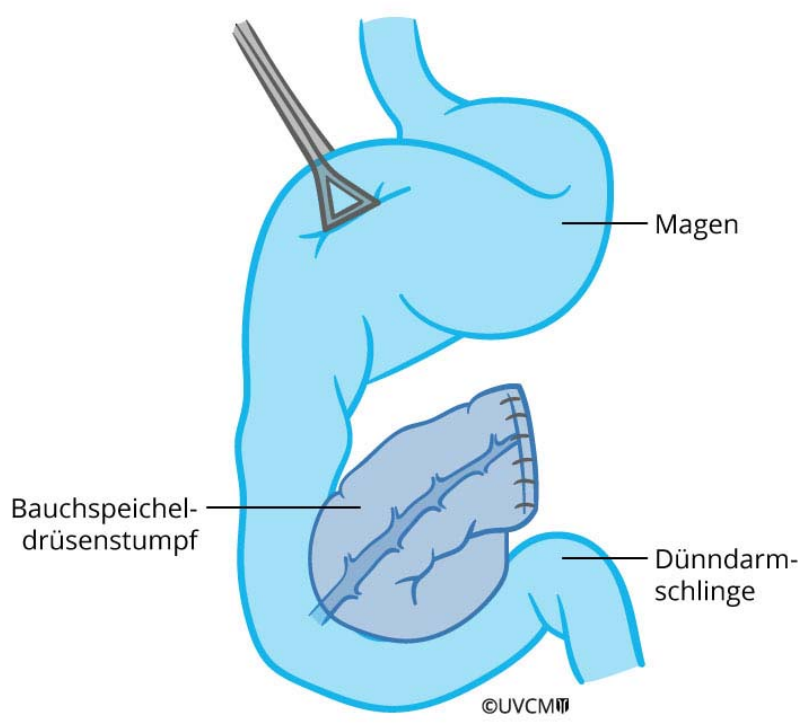


Abbildung 2: Korrelation der beobachteten Mortalitätsrate 2008-2012 und der jährlichen Fallzahl ([16]

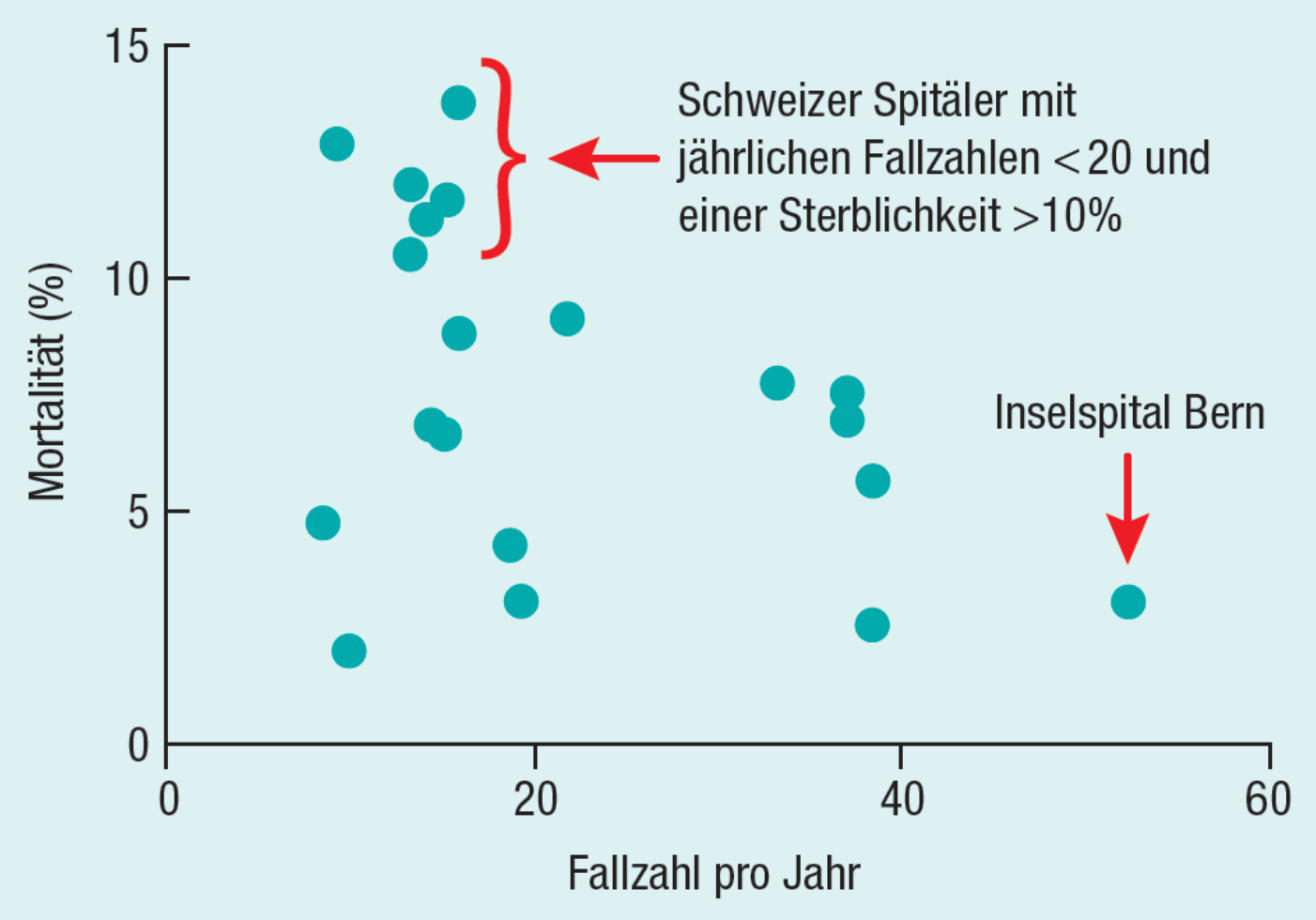


Abbildung 3: Irreversible Elektroporation - Exakte Platzierung der Ablationsnadel mittels computer-basierter Navigation

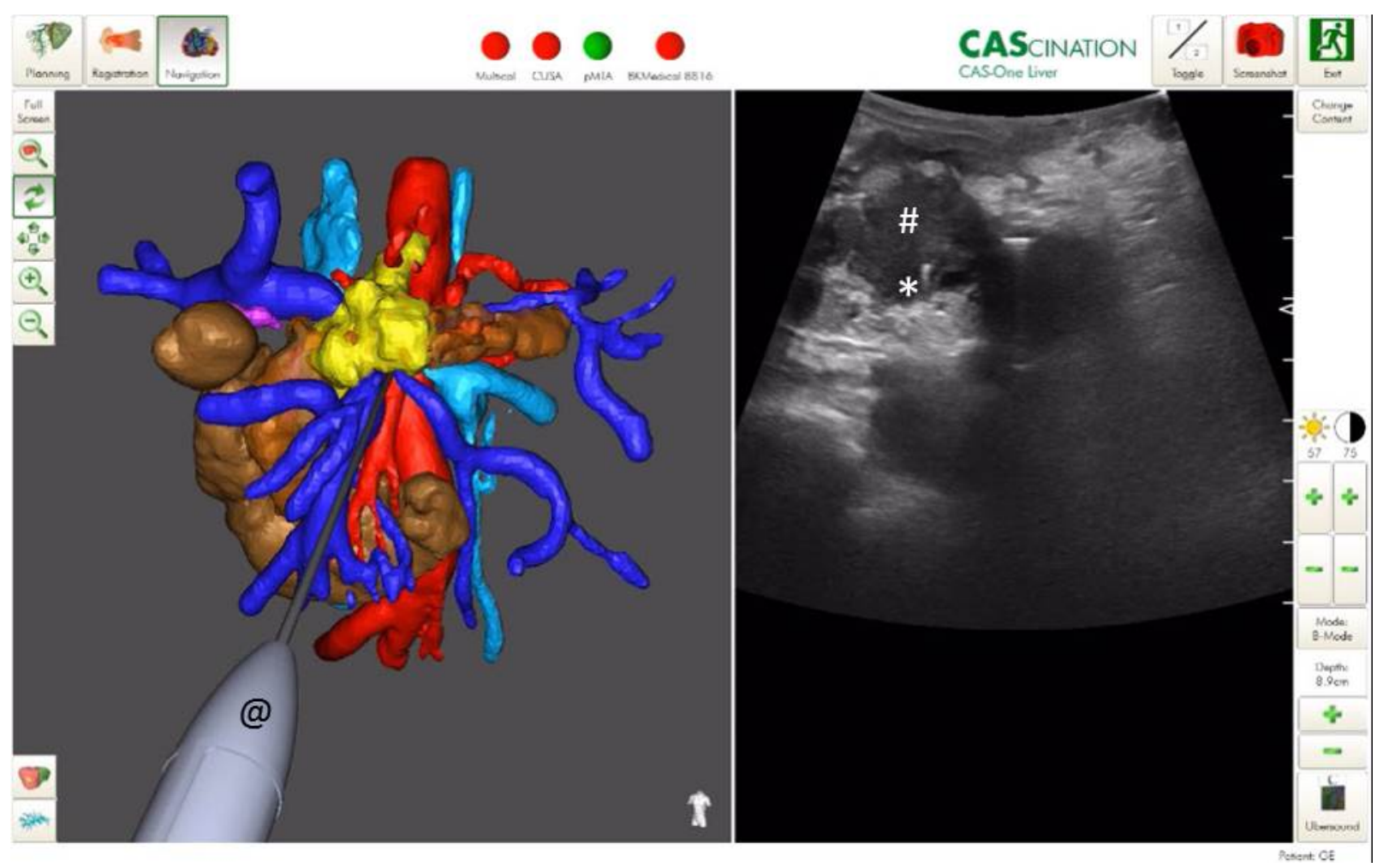

@: IRE-Nadel; \#: Pankreastumor; *: IRE-Nadel im Ultraschall; Gelb: lokal fortgeschrittenes Pankreaskarzinom mit Ummauerung des Truncus coeliacus; Blau: Pfortader mit Zuflüssen; Rot: Aorta mit Abgängen; Braun: Pankreas und Duodenum 
1. International Agency for Research on Cancer, World Health Organization. http://globocan.iarc.fr/ (last accessed 06.01.2016).

2. Siegel, R.L., K.D. Miller, and A. Jemal, Cancer statistics, 2015. CA Cancer J Clin, 2015. 65(1): p. 5-29.

3. Baxter, N.N., B.A. Whitson, and T.M. Tuttle, Trends in the treatment and outcome of pancreatic cancer in the United States. Ann Surg Oncol, 2007. 14(4): p. 1320-6.

4. $\quad$ Roland, C.L., et al., The Addition of Postoperative Chemotherapy is Associated with Improved Survival in Patients with Pancreatic Cancer Treated with Preoperative Therapy. Ann Surg Oncol, 2015. 22 Suppl 3: p. 1221-8.

5. $\quad$ Esposito, I., et al., Most pancreatic cancer resections are R1 resections. Ann Surg Oncol, 2008. 15(6): p. 1651-60.

6. $\quad$ Kleeff, J., et al., Pancreatic cancer. Nat Rev Dis Primers, 2016. 2: p. 16022.

7. Al-Hawary, M., Role of Imaging in Diagnosing and Staging Pancreatic Cancer. J Natl Compr Canc Netw, 2016. 14(5 Suppl): p. 678-80.

8. Halperin, D.M. and G.R. Varadhachary, Resectable, borderline resectable, and locally advanced pancreatic cancer: what does it matter? Curr Oncol Rep, 2014. 16(2): p. 366.

9. Barreto, S.G. and J.A. Windsor, Justifying vein resection with pancreatoduodenectomy. Lancet Oncol, 2016. 17(3): p. e118-24.

10. Riediger, H., et al., Postoperative morbidity and long-term survival after pancreaticoduodenectomy with superior mesenterico-portal vein resection. J Gastrointest Surg, 2006. 10(8): p. 1106-15.

11. Kato, H., et al., Clinical features and treatment outcome of borderline resectable pancreatic head/body cancer: a multi-institutional survey by the Japanese Society of Pancreatic Surgery. J Hepatobiliary Pancreat Sci, 2013. 20(6): p. 601-10.

12. Asbun, H.J., et al., When to perform a pancreatoduodenectomy in the absence of positive histology? A consensus statement by the International Study Group of Pancreatic Surgery. Surgery, 2014. 155(5): p. 887-92.

13. Worni, M., et al., Concomitant vascular reconstruction during pancreatectomy for malignant disease: a propensity score-adjusted, population-based trend analysis involving 10,206 patients. JAMA Surg, 2013. 148(4): p. 331-8.

14. Riviere, D., et al., Laparoscopic versus open distal pancreatectomy for pancreatic cancer. Cochrane Database Syst Rev, 2016. 4: p. CD011391.

15. Bencini, L., et al., Minimally invasive surgical approach to pancreatic malignancies. World J Gastrointest Oncol, 2015. 7(12): p. 411-21.

16. http://www.bag.admin.ch/hospital/index.html StichwortQualitätsindikatoren.

17. Finks, J.F., N.H. Osborne, and J.D. Birkmeyer, Trends in hospital volume and operative mortality for high-risk surgery. N Engl J Med, 2011. 364(22): p. 2128-37.

18. de Wilde, R.F., et al., Impact of nationwide centralization of pancreaticoduodenectomy on hospital mortality. Br J Surg, 2012.99(3): p. 404-10.

19. Gillen, S., et al., Preoperative/neoadjuvant therapy in pancreatic cancer: a systematic review and meta-analysis of response and resection percentages. PLoS Med, 2010. 7(4): p. e1000267.

20. Blazer, M., et al., Neoadjuvant modified (m) FOLFIRINOX for locally advanced unresectable (LAPC) and borderline resectable (BRPC) adenocarcinoma of the pancreas. Ann Surg Oncol, 2015. 22(4): p. 1153-9.

21. Frigerio, I., et al., Short term chemotherapy followed by radiofrequency ablation in stage III pancreatic cancer: results from a single center. J Hepatobiliary Pancreat Sci, 2013. 20(6): p. 574-7.

22. Martin, R.C., 2nd, et al., Irreversible electroporation therapy in the management of locally advanced pancreatic adenocarcinoma. J Am Coll Surg, 2012. 215(3): p. 361-9. 
23. Miller, L., J. Leor, and B. Rubinsky, Cancer cells ablation with irreversible electroporation. Technol Cancer Res Treat, 2005. 4(6): p. 699-705.

24. Maor, E., et al., The effect of irreversible electroporation on blood vessels. Technol Cancer Res Treat, 2007. 6(4): p. 307-12.

25. Martin, R.C., 2nd, et al., Treatment of 200 Locally Advanced (Stage III) Pancreatic Adenocarcinoma Patients With Irreversible Electroporation: Safety and Efficacy. Ann Surg, 2015. 262(3): p. 486-94.

26. Kluger, M.D., et al., Single-Institution Experience with Irreversible Electroporation for T4 Pancreatic Cancer: First 50 Patients. Ann Surg Oncol, 2015.

27. Martin, R.C., 2nd, et al., Irreversible electroporation in locally advanced pancreatic cancer: potential improved overall survival. Ann Surg Oncol, 2013. 20 Suppl 3: p. S443-9.

28. Banz, V.M., et al., Intraoperative image-guided navigation system: development and applicability in 65 patients undergoing liver surgery. Langenbecks Arch Surg, 2016. 401(4): p. 495-502. 


\section{Surgical and interventional treatment in pancreatic adenocarcinoma}

Only in a minority of $20 \%$ of patients with pancreatic cancer the disease is diagnosed at an locally limited stage, what allows for a R0 resection. This is associated with a 5year survival of 20 to $25 \%$.

But also in so called "borderline resectable" and "locally advanced" disease a surgical treatment may combined with intraoperative irreversible electroporation can be applied in selected cases and in the context of multimodal treatment, thereby improving prognosis.

In high-volume centers resection of tumors with limited venous involvement increases the number of $R O$ resections without significantly increasing morbidity and mortality. 